\title{
Transmisión transplacentaria de Neospora caninum en bovinos de cría de la Provincia de Corrientes, Argentina
}

\author{
Martínez, D. '; Jacobo, R. ${ }^{1}$; Cipolini, F.'; Martínez, I. ${ }^{1}$; Echaide, I. ${ }^{2}$
}

${ }^{1}$ Cátedra Enfermedades Infecciosas, Facultad de Ciencias Veterinarias, Universidad Nacional del Nordeste, Sargento Cabral 2139, Corrientes (3400), Argentina. Tel 379-4425753. Instituto Nacional de Tecnología

Agropecuaria (INTA), Rafaela (Santa Fe, Argentina).E-mail: demartinez@vet.unne.edu.ar.

\begin{abstract}
Resumen
Martínez, D.; Jacobo, R.; Cipolini, F.; Martínez, I.; Echaide, I.: Transmisión transplacentaria de Neospora caninum en bovinos de cría de la Provincia de Corrientes, Argentina. Rev. vet. 23: 1, 43-45, 2012. La neosporosis es una de las principales enfermedades causantes de abortos en bovinos. Su agente etiológico es Neospora caninum, protozoario Apicomplexa transmitido al ganado por vía transplacentaria y alimentos contaminados por deyecciones de distintas especies de cánidos, sus hospedadores definitivos. En el presente trabajo se evaluó la frecuencia de transmisión de $N$. caninum in utero y post partum en 56 vacas de raza Brahman y Brangus serológicamente positivas, así como en sus crías al nacimiento y prospectivamente hasta el año de edad, mediante las pruebas de enzimoinmunoensayo indirecto (ELISAi), cuyos resultados se expresaron en porcentaje de positividad (PP), e inmunofluorescencia indirecta. La proporción de animales positivos a neosporosis en las vacas y sus crías fue de $14,3 \%$ (8/56) y $17,9 \%(10 / 56)$ respectivamente. Cuarenta y seis vacas y sus terneros se clasificaron negativos a neosporosis (4,2 $\pm 3,8 \mathrm{PP}$, ELISAi). De los 10 terneros positivos, 8 eran hijos de madres positivas y de éstos sólo 5 fueron reactores positivos antes de ingerir calostro (51,6 $\pm 17,3$ PP, ELISAi). Los 3 terneros restantes alcanzaron un valor similar (59,0 $\pm 38,1$ PP, ELISAi). En el $62 \%$ de los terneros los niveles de anticuerpos se mantuvieron $\geq 25$ PP hasta los 6 meses de edad. Dos terneros nacidos con serología negativa seroconvirtieron a los nueve meses de edad.
\end{abstract}

Palabras clave: bovino de carne, neosporosis, diagnóstico serológico, Argentina.

\begin{abstract}
Martínez, D.; Jacobo, R.; Cipolini, F.; Martínez, I.; Echaide, I.: Transplacentary Neospora caninum transmission in breeding cattle from Corrientes Province, Argentina. Rev. vet. 23: 1, 43-45, 2012. Neosporosis is one of the most important diseases that causes reproductive loses in cattle. It is caused by Neospora caninum, Apicomplexa protozoarian that infects cattle due to consumption of contaminated foods with excrements of canidae, its definitive host, and by transplacentary way. In this work it was evaluated the frequency of transplacentary and after-born transmission of $N$. caninum in 56 positive Brahman and Brangus cows and their calves at birth and prospectively until one-year-old, through the indirect enzymeimmunoassay (ELISAi). Results were expressed as percentages of positivity (PP), and indirect immunefluorescence serological tests. The proportion of positive cows and calves were $14.3 \%(8 / 56)$ y $17.9 \%$ (10/56), respectively. Forty six cows and their calves were classified as neosporosisnegative (4.2 $\pm 3.8 \mathrm{PP}$, ELISAi). From 10 positive calves 8 were born from positive dams and from these only 5 were positive to serological tests before colostrums consumption $(51.6 \pm 17.3$ PP, ELISAi). The 3 remaining calves reached a similar value (59.0 \pm 38.1 PP, ELISAi). In 62\% of the calves the antibody levels kept on $\geq 25$ PP until 6 months-old. Two calves that were born serologically negative became positive at 9 months-old.
\end{abstract}

Key words: beef cattle, neosporosis, serologic diagnosis, Argentina.

\section{INTRODUCCIÓN}

La neosporosis es considerada una de las principales enfermedades causante de abortos en bovinos. Es provocada por Neospora caninum, protozoario Apicomplexa cuyo ciclo de vida depende de los cánidos,

Recibido: 22 marzo 2012 / Aceptado: 4 mayo 2012 sus hospedadores definitivos, y de distintas especies intermediarias entre los que se destacan los bovinos por su mayor susceptibilidad. El parásito fue identificado primero en perros ${ }^{1} \mathrm{y}$ posteriormente en fetos abortados de bovinos ${ }^{9}$.

El severo daño tisular causado por los taquizoítos de $N$. caninum en el placentoma o en diversos tejidos del feto provocan abortos, principalmente durante los 
primeros meses de gestación. Los taquizoítos alcanzarían la placenta después de infecciones ocurridas durante la gestación o la reactivación de quistes tisulares en hembras con infección crónica ${ }^{2,3}$. Cuando esta invasión ocurre después de la mitad de la gestación, se incrementan las posibilidades que el parásito se transmita por vía transplacentaria al feto, sin causar lesiones que impidan el nacimiento de un ternero infectado, aunque sin signos clínicos de neosporosis ${ }^{11}$.

Los valores de prevalencia de neosporosis en bovinos de cría frecuentemente son menores a los de bovinos lecheros, probablemente por el manejo requerido para cada tipo de producción. Sin embargo no se ha establecido claramente la susceptibilidad para sufrir abortos o transmisión transplacentaria de las distintas razas de bovinos.

El objetivo de este trabajo fue evaluar transmisión transplacentaria de $N$. caninum y ocurrencia de nuevos casos durante el primer año de vida en bovinos de raza Brahman y Brangus en un rodeo de cría de la Provincia de Corrientes, Argentina.

\section{MATERIAL Y MÉTODOS}

Bovinos. El estudio prospectivo en terneros se realizó en un rodeo de cría cerrado, ubicado en el Departamento de Concepción, Provincia de Corrientes, Argentina. La edad promedio de las hembras al iniciar el trabajo fue de 5 años ( 3 a 7 años); el porcentaje de preñez histórico del rodeo era de $70 \%$, con una tasa de abortos del $1 \%$, estimada sobre bovinos con preñez confirmada por palpación rectal. Las hembras preñadas próximas al parto fueron aisladas en corrales para facilitar la obtención de muestras de sangre de los terneros antes de que ingirieran calostro.

Muestras. Se tomaron por única vez muestras de suero de 56 vacas de raza Brahman y Brangus, previo al parto, por punción de la vena yugular. Para identificar a los terneros que se hubiesen infectado durante la gestación, se tomaron muestras de sangre inmediatamente después del parto, en un período preestablecido de 6 horas, para tratar de evitar la ingestión de calostro, obteniéndose en total ocho muestras periódicas (M1 a M8) hasta el año de edad. M1 se tomó durante los meses de agosto y septiembre de 2009. Para evaluar las fluctuaciones de los niveles de anticuerpos en los terneros, las restantes M2 a M8 se obtuvieron con una periodicidad promedio de 36 días. En todos los casos las muestras de sangre se obtuvieron por punción de la vena yugular y los sueros se almacenaron a $-20^{\circ} \mathrm{C}$ hasta su utilización.

Prueba de glutaraldehído. Se realizó según una técnica previamente descrita ${ }^{7}$, para evaluar si los terneros habían ingerido calostro. Se consideró que habían ingerido calostro los terneros cuyos sueros coagularon dentro de los $30 \mathrm{~min}$ de agregado el glutaraldehido.

Tests de enzimoinmunoensayo e inmunofluorescencia indirecta. La presencia de anticuerpos específicos contra $N$. caninum en todas las muestras de suero
Tabla 1. Evolución del promedio de PP (ELISAi) de terneros que ingirieron calostro antes de la muestra 1.

\begin{tabular}{ccccc}
\hline $\begin{array}{c}\text { identificación } \\
\text { del ternero }\end{array}$ & $\begin{array}{c}\text { estatus } \\
\text { madre }\end{array}$ & $\begin{array}{c}0-6 \\
\text { horas }\end{array}$ & $\begin{array}{c}3-4 \\
\text { meses }\end{array}$ & $\begin{array}{c}4-9 \\
\text { meses }\end{array}$ \\
\hline 1 & + & 37 & 28 & 27 \\
2 & + & 103 & 52 & 23 \\
3 & + & 37 & 31 & 16 \\
4 & - & 26 & 6 & 6 \\
5 & - & 30 & 4 & 8 \\
\hline
\end{tabular}

Los números indican, para cada uno de los tres períodos muestreados (nacimiento a 9 meses de edad), los niveles medios de porcentaje de positividad (PP) para anticuerpos contra $N$. caninum obtenidos mediante ELISAi, alcanzados por los 5 terneros que ingirieron calostro antes de M1.

se determinó mediante un enzimoinmunoensayo indirecto (ELISAi) e inmunofluorescencia indirecta (IFI) ${ }^{4}$. Los sueros se diluyeron 1/100 antes de ser usados en cada prueba. Para ELISAi se procedió a realizar la lectura por duplicado para cada muestra. Los resultados del ELISAi se expresaron como promedio del porcentaje de positividad (PP) entre las dos repeticiones del test para cada muestra. Las vacas cuyos sueros arrojaron títulos de anticuerpos $\geq 25 \%$ (ELISAi) o $\geq 1 / 100$ (IFI) se consideraron infectadas. Los sueros de terneros se analizaron mediante ELISAi y los resultados se interpretaron en relación a los PP obtenidos para la M1 de los terneros nacidos de madres negativas.

Estadísticas. Dado que la cantidad de animales incluidos en el estudio fue insuficiente para estimar la prevalencia de la enfermedad, mediante estadísticas descriptivas se estimó la proporción de animales positivos a neosporosis. Para el test de ELISAi, los PP de cada suero corresponden al promedio obtenido para cada muestra analizada por duplicado. Se estimaron las medias aritméticas y los desvíos estándares de los títulos de anticuerpos de hembras y crías, positivos y negativos.

\section{RESULTADOS}

La prevalencia de neosporosis en el grupo de 56 hembras adultas fue de 14,3\% (8/56). Los títulos alcanzados por los ejemplares positivos a ELISAi (media \pm desvío estándar) en 6 vacas reactoras fue de 40,0 $\pm 13,6$ PP (muestras preparto). Las dos vacas positivas restantes se identificaron mediante IFI.

Los ocho terneros nacidos de vacas infectadas fueron reactores serológicos positivos, y sólo 3 de ellos habrían ingerido calostro de acuerdo con la prueba del glutaraldehído. Los títulos alcanzados por los sueros de los 5 terneros que no habían ingerido calostro (ELISAi) fueron de 51,6 \pm 17,3 PP, y para los tres restantes de $59 \pm 38,1 \mathrm{PP}$ en la M1. Estos niveles de anticuerpos se mantuvieron $\geq 25$ PP hasta los 6 meses de edad en el $62 \%$ de los terneros. 
De las 48 hembras cuyos sueros fueron negativos a ELISAi, 46 parieron terneros con serología negativa con títulos de anticuerpos promedio de 4,2 $\pm 3,8 \mathrm{PP}$. Las dos restantes parieron terneros con serología positiva y un título promedio de $28 \pm 2,8$ PP (M1) en ELISA pero negativos a la prueba de IFI. El período de extinción de los anticuerpos calostrales, en los 5 terneros positivos en M1 fue observado a los 3 meses de vida. El perfil serológico de los terneros hijos de madres positivas fue diferente al de los terneros hijos de madres negativas (Tabla 1).

Se detectaron dos infecciones postnatales en dos teneros hijos de vacas negativas, los que seroconvirtieron a los 9 meses de edad.

\section{DISCUSIÓN}

La proporción de animales positivos a neosporosis de $14,3 \%$ hallada en este trabajo sería esperable en función de la prevalencia de $16,8 \%$ encontrada en otros rodeos de cría de Corrientes ${ }^{5} \mathrm{y}$ menor a la registrada en bovinos lecheros de Argentina ${ }^{4}$. Los tres terneros positivos en M1 que previamente habían ingerido calostro mostraron un perfil serológico similar al de aquellos terneros infectados in utero, y aunque no fue posible aseverarlo fueron considerados como infecciones transplacentarias.

Si bien el nivel de transmisión transplacentaria alcanzó el 100\%, habría que considerarlo como una tendencia ya que el número de muestras involucradas en este estudio fue limitado. Aunque algunos investigadores también encontraron prevalencia del $100 \%{ }^{10}$, otros reportaron valores de transmisión transplacentaria tan dispares como $37,1 \%{ }^{8}$ y $93 \%{ }^{6}$. Tales diferencias estarían asociadas a la edad reproductiva de las hembras, correspondiendo los valores más elevados a las vaquillonas ${ }^{10}$. Este resultado es coherente con las observaciones obtenidas en rodeos lecheros, que sugieren que la transmisión transplacentaria sería más frecuente y eficiente que la horizontal ${ }^{6}$.

La infección de dos terneros después del nacimiento sugiere la presencia de fuentes de infección en el rodeo, representadas probablemente por alimentos o agua contaminada por ooquistes de $N$. caninum. La falta de correspondencia detectada entre dos vacas con serología negativa y sus respectivos terneros con serología positiva a ELISAi, puede responder a posibles reacciones inespecíficas, lo que contrastó con el resultado negativo obtenido mediante IFI, coincidente con el estatus de las madres. Alternativamente podría coincidir con la existencia de niveles fluctuantes de anticuerpos como se ha observado en estudios prospectivos anteriores.

En conclusión, los resultados aseveran que la vía de transmisión transplacentaria de $N$. caninum es importante en bovinos Brahman y Brangus, y que no sería menor la susceptibilidad a tal infección en estas razas. Sin embargo un nuevo diseño experimental donde se incluyan otras pruebas de diagnóstico directo y un ma- yor número de animales, deberá llevarse a cabo para confirmar estas observaciones.

Agradecimientos. Por su valioso aporte, a los MV Carlos Storani y Arnaldo Ragazzi, integrantes del grupo de trabajo de la Facultad de Ciencias Veterinarias UNNE y a la MV Beatriz Valentini del INTA Rafaela.

\section{REFERENCIAS}

1. Bjerkas I, Mohn SF, Presthus J. 1984. Unidentified cystforming sporozoon causing encephalomyelitis and myositis in dogs. $Z$ Parasitenkd 70: 271-274.

2. Dubey JP, Lindsay DS. 1999. Neosporosis in cattle. Biology and economic impact. J Am Vet Med Assoc 214: 11601163.

3. Dubey JP. 2003. Neosporosis in cattle. J Parasitol 89: 4256.

4. Echaide I, Valentini B, Torioni S. 2002. Neosporosis bovina: análisis seroepidemiológico de un hato lechero mediante IFA y ELISA. Memorias XIV Reun Cientif AAVLD, Villa Gral. Belgrano (Córdoba, Argentina), p. 01.

5. Moore DP, Odeón AC, Venturini MC, Späth E, Leunda MR, Draghi MG, Cetra B, Campero CM. 2000. Seroprevalencia a Neospora caninum en terneros de cría del sur de Corrientes. Memorias III Congr Arg Parasitol, Mar del Plata (Argentina), p. 4413.

6. Moré G, Bacigalupe D, Basso W, Rambeaud M, Beltrame F, Ramirez B, Venturini MC, Venturini L. 2009. Frequency of horizontal and vertical transmission for Sarcocystis cruzi and Neospora caninum in dairy cattle. Vet Parasitol 160: 51-54.

7. Sandholm M. 1974. A preliminary report of a rapid method for the demonstration of abnormal gamma globulin levels in bovine whole blood. Res Vet Sci 17: 32-35.

8. Schares G, Peters M, Wurm R, Bärwald A, Conraths FJ. 1998. The efficiency of vertical transmission of Neospora caninum in dairy cattle analyzed by serological techniques. Vet Parasitol 80: 87-98.

9. Thilsted JP, Dubey JP. 1989. Neosporosis-like abortions in a herd of dairy cattle. J Vet Diagn Invest 1: 205-209.

10. Thurmond M, Hietala S, Blanchard PC. 1997. Herd-based diagnosis of Neospora caninum induced endemic and epidemic abortion in cows and evidence for congenital and postnatal transmission. J Vet Diagn Invest 9: 44-49.

11. Williams DJ, Guy CS, McGarry JW, Guy F, Tasker L, Smith RF, MacEachern K, Cripps P, Kelly DF, Trees AJ. 2000. Neospora caninum- associated abortion in cattle: the time of experimentally-induced parasitaemia during gestation determines foetal survival. Parasitol 121: 347-358. 